\title{
The implications of terminal $6 q$ deletion syndrome: determining appropriate anticipatory guidance, evaluation, and management: a case report and analysis of published literature
}

\begin{abstract}
Terminal $6 \mathrm{q}$ deletions have been infrequently described and have a variable phenotype. The constellation of symptoms described includes characteristic facies, developmental delay, growth delay, urogenital abnormalities, brain malformations, and cardiovascular malformations. Identification of a terminal $6 \mathrm{q}$ deletion should prompt an appropriate evaluation as well as thorough anticipatory guidance for the family. Here we describe an additional case of a terminal $6 \mathrm{q}$ deletion and review all previously reported cases.
\end{abstract}

Volume 5 Issue I - 2018

Rohit S Loomba MD
Cincinnati Children's Hospital Medical Center, USA

Correspondence: Rohit S Loomba MD, Cincinnati Children's Hospital Medical Center, 3333 Burnet Avenue, Cincinnati, $\mathrm{OH}$, 45202, USA, Email loomba.rohit@gmail.com

Received: October 16, 2017 | Published: January 19, 2018

\section{Introduction}

Terminal 6q deletions (those occurring from q25.0 onwards) have been infrequently described in the literature but do appear to represent a syndrome with some common phenotypic features. Recognition of terminal 6q deletion syndrome is important as this allows for proper anticipatory guidance to be provided to patients and their families, evaluation for associated abnormalities, and the implementation of appropriate management strategies. While this holds true, there is no standardized protocol for the evaluation of management of these children due to its rarity. Case reports of terminal $6 \mathrm{q}$ deletion syndrome exist and offer a majority of available data, demonstrating the variability not only in the phenotype of patients but also the evaluation and management of these patients. We describe here a case of a young boy noted to have a terminal $6 \mathrm{q}$ deletion syndrome in infancy and analyze this alongside data extracted from previous case reports of terminal $6 \mathrm{q}$ deletions in children and adults to describe the frequency of phenotypic characteristics, describe the most common phenotype, and determine appropriate evaluation and management strategies for those noted to have terminal $6 \mathrm{q}$ deletions.

\section{Methods}

A systematic review of the literature was performed to identify manuscripts describing terminal $6 \mathrm{q}$ deletions. This was a newly conducted review with no previous review protocol having been established for it. The aim of the study was identified as being that of describing the phenotypic characteristics of both children and adults with terminal $6 \mathrm{q}$ deletions and characterizing the evaluation of these patients.

\section{Search strategy}

Manuscripts were identified using electronic databases including PubMed, EMBASE, and Ovid which were queried using the following search terms: "6q deletion" and "6q monosomy". No specific restriction on year of publication was used. Resulting studies were then screened by title and abstract with manuscripts describing deletions on chromosome $6 \mathrm{q}$ subsequently being retrieved in their entirety. References of these were hand searched for additional relevant manuscripts. No direct with manuscript authors was required to obtain full text manuscripts. These full text manuscripts were then reviewed by two of the authors. Published manuscripts available in full text were included in this review if they presented cases of $6 q$ deletion with patient level data, were in English, and clearly identified a genetic deletion incorporating the terminal portion of $6 \mathrm{q}$ (defined as q25 to qter for this study).

\section{Data extraction}

Next, data regarding several clinical features of terminal $6 \mathrm{q}$ deletion were extracted from the manuscripts identified for inclusion. This data was extracted independently with use of a data collection form. Clinical features mentioned to be present or absent in the text or tables of the manuscripts were recorded as such. If no information was available about certain clinical features then this was designated separately. Authors of included studies were not contacted for additional data.

\section{Bias analysis}

Since all manuscripts identified for this study were case reports or case series no quantification of specific bias could be performed. Some studies described the same clinical features in a variety of ways and these were recorded as a single clinical feature during data extraction. For example, some studies described a "broad nasal tip" while others described a "bulbous nose". These were both treated as representing the same clinical feature and recorded as "broad nasal tip" in this review. Similarly, some studies described "narrow palpebral fissures" while others described "short palpebral fissures" both of which were recorded as "short palpebral fissures" in this review.

\section{Data analysis}

Numeric data is presented as means with standard deviations or medians with ranges. Categorical data is presented as frequencies 
with absolute numbers as well as percentages. Analysis was begun with a Pearson correlation of all binary clinical features to determine associations between individual features. A chi-square analysis was then done to determine whether particular clinical features were more likely in males and females. An independent samples T-test was then performed to determine the effect of deletion size on particular clinical features. P-values of (insert less than or equal to sign here) 0.05 were considered statistically significant. All analysis was done using SPSS statistical software, version 20.0 (Chicago, IL)

\section{Case report}

We present the case of an infant born at 38 weeks gestation in a twin gestation to a G6P5 mother at an outside institution. The pregnancy itself was uncomplicated and the mother's medications during pregnancy were fluodrocortisone acetate for palpitations and syncope as well as acetylsalicylic acid due to history of a previous stroke. Prenatal care was complete and prenatal ultrasounds had demonstrated what appeared to be normal fetal structures although the mother shares that there was mention of some concern of the heart but a fetal echocardiogram was not obtained. Maternal serum screening was normal. Delivery was via $\mathrm{C}$-section due to transverse lie but was otherwise uncomplicated. The child was taken to the nursery where he spent a day and a half before being taken to the neonatal intensive care unit due to increased work of breathing. He was placed on supplemental oxygen and had an echocardiogram done which demonstrated a moderate sized ventricular septal defect and a patent ductus arteriosus with a small shunt. Mild mitral valve insufficiency was also present. His respiratory distress improved without additional intervention and with weans to room air and he was discharged home after 5 days.

At approximately 10 days of life, the baby began to have decreased oral intake, decreased urine output, and tachypnea. At 11 days of life he was brought to the emergency department at our institution where he was found to be mildly dehydrated and with signs and symptoms consistent with heart failure secondary to pulmonary overcirculation. On physical examination he was noted to have a widened nasal bridge, a broad nasal tip, low-set ears, bilateral preauricular pits, and cryptorchidism. Weight was $2.50 \mathrm{~kg}$ ( $2^{\text {nd }}$ percentile $)$, height was $48.30 \mathrm{~cm}\left(20^{\text {th }}\right.$ percentile). He was started on anticongestive therapy consisting of furosemide and captopril which did result in symptom relief as well as decrease in his brain natriuretic peptide values from 1120 on initial evaluation in the emergency room to 60 after approximately one month of treatment. The ventricular septal defect closed spontaneously by 1year of age. At approximately 8 months of age the patient began developing periods of unresponsiveness and hypoxia which were thought to be concerning for seizures. He also had an episode of uncontrolled jerking of all his extremities with concomitant hypoxia. Although EEGs were obtained and were normal the child was started on levetiracetam with improvement in the frequency of such events. A genetic microarray was obtained in the first month of life and demonstrated a terminal $6 \mathrm{q}$ deletion.

\section{Results}

A total of 683 unique manuscripts were identified by initial electronic search using the databases identified above. Of these, 119 were deemed relevant after review of titles and abstracts with full text manuscripts obtained for 102 of these. After review of the full text manuscripts, 35 studies were identified for inclusion in the review. ${ }^{1-35}$ This resulted in 72 cases reported in the literature that when combined with the case presented below leads to 73 cases presented in this review. Of those in this cohort, 7\% were prenatally diagnosed. The median age of postnatal diagnosis was 14 months with a range of 0.5 to 444 months. Over half of the cohort (51\%) was male. De novo deletions were found in $85 \%$ of cases with a median deletion size of 8 megabase pairs with a range of 0.3 to 203.4. Deletions were detected by fluorescence in situ hybridization (FISH) in 57\% cases, karyotype in $22 \%$, and array in $21 \%$. Parental consanguinity was present in $5 \%$ of cases.

Facial dysmorphisms included, but were not limited to, the following: short palpebral fissures in $24 \%$, prominent nasal bridge in $69 \%$, broad nasal tip in $59 \%$, downturned corners of the mouth in $14 \%$, and ear abnormalities in 78\%. Eye or vision abnormalities were noted in $58 \%$. Just a little of a third of the cohort (34\%) had a short neck and nearly half (46\%) had microcephaly. Cranial suture abnormalities were documented in $20 \%$ of patients. Other malformations included urogenital abnormalities in $27 \%$ and extremity abnormalities in $55 \%$. Developmental delay of some variety was noted in almost all patients. An overwhelming majority (90\%) of those in the cohort were found to have a degree of intellectual disability. Motor delay was noted in $97 \%$ and speech delay in $68 \%$. Motor delay was present in $97 \%$. Seizures were noted in $57 \%$. Brain imaging was obtained for $78 \%$ of patients with magnetic resonance imaging making up $79 \%$ of these tests. Brain imaging demonstrated hydrocephalus (33\%), a pons abnormality in $5 \%$, grey/white matter abnormalities in $9 \%$, corpus collosum abnormalities in $46 \%$ of patients.

Cardiac abnormalities were noted in $30 \%$ of patients. Atrial septal defect was the most common, being found in $36 \%$ of patients with a cardiac abnormality. Ventricular septal defect was found in $23 \%$. Other cardiac lesions noted included Tetralogy of Fallot, atrioventricular septal defect, and cor triatriatum. Also worth mention are cyanotic spells that were noted in $15 \%$ of this cohort. These did not demonstrate any statistically significant association with the presence of cardiac abnormalities or seizures. There are very few differences noted in phenotype between males and females. Males were more likely to have de novo mutations $(\mathrm{p}=0.021)$ while females were more likely to have downturned corners of the mouth $(\mathrm{p}=0.036)$. Deletion size does seem to impact the phenotypic expression. Larger deletions were found in those with prominent nasal bridge $(p=0.025)$, ear abnormalities $(p=0.038)$, and corpus collosum abnormalities $(\mathrm{p}=0.007)$. Those with Cranial suture abnormalities tended to have smaller deletions than those without $(\mathrm{p}=0.009)$. Not enough data was present to compare deletion size in those with mental retardation, speech delay, or motor delay. Correlation analysis run between all the studied characteristics determined the following phenotype to be the most common: broad nasal bridge, ear abnormalities, broad nasal tip, microcephaly, mental retardation, motor delay, and urogenital abnormalities.

\section{Discussion}

Terminal $6 \mathrm{q}$ deletions are relatively infrequent and thus there is limited data available. Additionally, there seems to be significant phenotypic variability which further complicates characterizing this group of patients. This poses difficulty to those providing care for these patients as there is a lack of guidance regarding what abnormalities patients should be evaluated for and what anticipatory guidance patients and other care providers should provide. Facial dysmorphism in terminal $6 \mathrm{q}$ deletion syndrome consist of short and downslanting palpebral fissures, flat and broad nasal bridge, broad nasal tip, and downturned corners of the mouth. Ear abnormalities are not infrequent and include low-set, posteriorly rotated ears. 
Many patients have also been reported to have preauricular pits. Eye abnormalities, other than those pertaining to the palpebral fissures, include strabismus and corneal abnormalities. Cranial malformations common in those with terminal $6 \mathrm{q}$ deletion syndrome and include overriding sutures, widened anterior fontanel, and microcephaly. Brain abnormalities are also frequently encountered and include hydrocephalus, pons abnormalities, grey/white matter abnormalities, and corpus collosum abnormalities. The spectrum of corpus collosum abnormalities is quite vast from mild dysgenesis to complete agenesis.

Seizures are also frequently noted in children with terminal $6 \mathrm{q}$ deletion syndrome. These may or may not be associated with abnormalities noted on brain imaging. Perhaps more interesting is that some cases have described seizure like episodes that are not associated with EEG abnormalities. In some of these cases the children were started on antiepileptic drugs with improvement in their symptoms while others were followed and some eventually had EEG changes and were started on antiepileptic drugs. Seizures can be very frequent, particularly in early childhood, with patients having 4-5 seizures a day. Seizures refractory to single agent therapy also appear to be common. Developmental delay is almost uniformly present. Intellectual disability is not uncommon but appears to be mild to moderate in most cases. Speech delay is also present with most cases being worse from an expressive rather than receptive nature. Growth delay with poor weight gain is also noted in early childhood. These delays may or may not be associated with hypotonia. Cardiac abnormalities are variable and most commonly consist of septal defects. Of interest are cyanotic episodes that occur in children with terminal $6 \mathrm{q}$ deletion which in the setting of cardiac abnormalities may be attributed to these abnormalities. In reality, however, most of these lesions are septal defects in the setting of subsystemic right ventricular pressures such that these would not have a reason to lead to right to left shunting and cyanosis. Thus, cyanotic episodes must not be automatically attributed to cardiac pathology. Other etiologies such as normal changes in vasomotor reactivity to environmental stressors, reflux, seizures, and upper respiratory infections should not be ignored.

The most frequent phenotype consists of broad nasal bridge, ear abnormalities, broad nasal tip, microcephaly, intellectual disability, motor delay, and urogenital abnormalities. This constellation of symptoms should thus raise suspicion for terminal $6 \mathrm{q}$ deletions and evaluation by microarray or FISH. If a terminal $6 \mathrm{q}$ deletion is noted the child should undergo an ophthalmologic examination and also have brain imaging, preferably magnetic resonance imaging. A thorough genital examination should be done as well and if testicles cannot be palpated in the scrotal sacs of males then abdominal ultrasonography should be obtained to identify their location. There must always be vigilance for seizures. Abnormal limb movements or repetitive, acute changes in interaction should raise suspicion for seizures. An EEG should be obtained but a normal EEG does not preclude these episodes from being seizures. Repeat EEG may be warranted, particularly, when the children are older if they continue to have episodes. Previous reports have described antiepileptics aborting abnormal movements in those with normal EEG so it would not be unreasonable to initiate such treatment if these movements are frequent. Because of the frequent developmental delay it is of utmost importance that all necessary therapy is arranged for early in life so as to ensure the best developmental outcome. Evaluation for physical therapy, occupational therapy, speech therapy all should be obtained early in childhood.

\section{Conclusion}

Particular constellations of syndromes should raise the suspicion for terminal $6 \mathrm{q}$ deletions and these patients should be evaluated for a series of physical malformations and clinical sequelae which include facial dysmorphism, developmental delay, cardiac abnormalities, brain abnormalities, cardiac abnormalities, and urogenital abnormalities. Once diagnosis is made by microarray or FISH, it is necessary to take a multidisciplinary approach in further evaluation and management.

\section{Acknowledgements}

None.

\section{Conflict of interest}

Author declares that there is no conflict of interest.

\section{References}

1. Abu-Amero KK, Hellani A, Salih MA, et al. Ophthalmologic abnormalities in a de novo terminal $6 \mathrm{q}$ deletion. Ophthalmic genet. 2010;31(1):1-11.

2. Backx L, Fryns JP, Marcelis C, et al. Haploinsufficiency of the gene Quaking $(\mathrm{QKI})$ is associated with the $6 \mathrm{q}$ terminal deletion syndrome. Am J Med Genet A. 2010;152A(2):319-326.

3. Bartoshesky L, Lewis MB, Pashayan HM. Developmental abnormalities associated with long arm deletion of chromosome No. 6. Clin genet. 1978;13(1):68-71

4. Bertini V, De Vito $\mathrm{G}$, Costa $\mathrm{R}$, et al. Isolated $6 \mathrm{q}$ terminal deletions: an emerging new syndrome. Am J Med Genet A. 2006;140(1):74-81.

5. Caselli R, Mencarelli MA, Papa FT, et al. A 2.6 Mb deletion of 6q24.3-25.1 in a patient with growth failure, cardiac septal defect, thin upperlip and asymmetric dysmorphic ears. Eur J Med Genet. 2007;50(4):315-321.

6. Chen CP, Chen M, Chen CY, et al. Prenatal diagnosis and molecular cytogenetic characterization of de novo pure partial trisomy $6 p$ associated with microcephaly, craniosynostosis and abnormal maternal serum biochemistry. Gene. 2014;536(2):425-429.

7. Choy KW, Chan LW, Tang MH, et al. Prenatal findings and delineation of de novo concurrent partial trisomy $7 \mathrm{q}(7 \mathrm{q} 31.2$--> qter) and partial monosomy $6 \mathrm{q}(6 \mathrm{q} 26$--> qter) by high-resolution array CGH. J Matern Fetal Neonatal Med. 2009;22(11):1014-1020.

8. Conti V, Carabalona A, Pallesi-Pocachard E, et al. Periventricular heterotopia in $6 \mathrm{q}$ terminal deletion syndrome: role of the C6orf70 gene. Brain. 2013;136(Pt 11):3378-3394.

9. Eash D, Waggoner D, Chung J, et al. Calibration of $6 \mathrm{q}$ subtelomere deletions to define genotype/phenotype correlations. Clin genet. 2005;67(5):396-403.

10. Elia M, Striano P, Fichera M, et al. $6 \mathrm{q}$ terminal deletion syndrome associated with a distinctive EEG and clinical pattern: a report of five cases. Epilepsia. 2006;47(5):830-838.

11. Evers LJ, Schrander-Stumpel CT, Engelen JJ, et al. Deletion of the long arm of chromosome 6: two new patients and literature review. Clin genet. 1996;50(3):138-144.

12. Fryns JP, Bettens W, Van den Berghe H. Distal deletion of the long arm of chromosome 6: a specific phenotype? Am J Med Genet. 1986;24(1):175-178.

13. Gerber JC, Neuhann TM, Tyshchenko N, et al. Expanding the clinical and neuroradiological phenotype of $6 \mathrm{q} 27$ microdeletion: olfactory bulb aplasia and anosmia. Am J Med Genet A. 2011;155A(8):1981-1986. 
14. Hopkin RJ, Schorry E, Bofinger M, et al. New insights into the phenotypes of 6q deletions. Am J Med Genet. 1997;70(4):377-386.

15. Koh S, Boles RG. Cerebral aqueductal stenosis as a presentation of deletion 6q25-qter. Clin genet. 1998;53(4):317-318.

16. Lee JY, Cho YH, Hallford G. Delineation of subtelomeric deletion of the long arm of chromosome 6. Ann Hum Genet. 2011;75(6):755-764.

17. McLeod DR, Fowlow SB, Robertson A, et al. Chromosome 6q deletions: a report of two additional cases and a review of the literature. Am J Med Genet. 1990;35(1):79-84.

18. Meloni VA, Guilherme RS, Oliveira MM, et al. Cytogenomic delineation and clinical follow-up of two siblings with an $8.5 \mathrm{Mb}$ 6q24.2-q25.2 deletion inherited from a paternal insertion. Am J Med Genet A. 2014;164A(9):2378-2384.

19. Meng J, Fujita H, Nagahara N, et al. Two patients with chromosome $6 \mathrm{q}$ terminal deletions with breakpoints at q24.3 and q25.3. Am J Med Genet. 1992;43(4):747-750.

20. Nagamani SC, Erez A, Eng C, et al. Interstitial deletion of 6q25.2-q25.3: a novel microdeletion syndrome associated with microcephaly, developmental delay, dysmorphic features and hearing loss. Eur J Hum Genet. 2009; 17(5):573-581.

21. Nair S, Varghese R, Hashim S, et al. Dysmorphic features and congenital heart disease in chromosome $6 \mathrm{q}$ deletion: A short report. Indian J Hum Genet. 2012;18(1):127-129.

22. Nowaczyk MJ, Carter MT, Xu J, et al. Paternal deletion 6q24.3: a new congenital anomaly syndrome associated with intrauterine growth failure, early developmental delay and characteristic facial appearance. Am J Med Genet A 2008;146A(3):354-360.

23. Oliveira-Duarte MH, Martelli-Soares LR, Sarquis-Cintra T, Machado ML, Lison MP (1990) Distal monosomy of the long arm of chromosome 6 (6q25----6qter) inherited by maternal translocation $\mathrm{t}(6 \mathrm{q} ; 17 \mathrm{q})$. Ann Genet 33(1):56-59.

24. Pirola B, Bortotto L, Giglio S, et al. Agenesis of the corpus callosum with Probst bundles owing to haploinsufficiency for a gene in an $8 \mathrm{cM}$ region of 6q25. J Med Genet. 1998;35(12):1031-1033.
25. Rivas F, Ruiz C, Rivera H, et al. De novo del(6)(q25) associated with macular degeneration. Ann Genet. 1986;29(1): 42-44.

26. Rubtsov N, Senger G, Kuzcera H, et al. Interstitial deletion of chromosome 6q: precise definition of the breakpoints by microdissection, DNA amplification, and reverse painting. Hum Genet. 1996;97(6):705-709.

27. Shen-Schwarz S, Hill LM, Surti U, et al. Deletion of terminal portion of 6q: report of a case with unusual malformations. Am J Med Genet. 1989;32(1):81-86.

28. Stevens CA, Fineman RM, Breg WR, et al. Report of two cases of distal deletion of the long arm of chromosome 6. Am J Med Genet. 1988;29(4):807-814.

29. Stevenson DA, Brothman AR, Carey JC, et al. 6q subtelomeric deletion: is there a recognizable syndrome? Clin Dysmorphol. 2004;13(2):103-106.

30. Striano P, Malacarne M, Cavani S, et al. Clinical phenotype and molecular characterization of $6 \mathrm{q}$ terminal deletion syndrome: Five new cases. Am J Med Genet A. 2006;140(18):1944-1949.

31. Su PH, Chen JY, Chen SJ, et al. Terminal deletion of chromosome $6 \mathrm{q}$ Pediatrics and neonatology. 2008;49(3):88-93.

32. Sukumar S, Wang S, Hoang K, et al. Subtle overlapping deletions in the terminal region of chromosome 6q24.2-q26: three cases studied using FISH. Am J Med Genet. 1999;87(1):17-22.

33. Tanteles GA, Yates K, Martin K, et al. Relatively mild phenotype in a patient with interstitial 6q24.3-q25.2 deletion. Clin Dysmorphol. 2007;16(2):101-104.

34. Titomanlio L, Giurgea I, Baumann C, et al. A locus for sacral/anorectal malformations maps to $6 \mathrm{q} 25.3$ in a $0.3 \mathrm{Mb}$ interval region. Eur J Hum Genet. 2006;14:971-974.

35. Valtat C, Galliano D, Mettey R, et al. Monosomy 6q: report on four new cases. Clin genet. 1992;41(3):159-166. 